\title{
Atomic and molecular gas in the starburst galaxy NGC $4945^{\star, \star \star}$
}

\author{
M. Ott ${ }^{1}$, J. B. Whiteoak ${ }^{2}$, C. Henkel ${ }^{1}$, and R. Wielebinski ${ }^{1}$ \\ 1 Max-Planck-Institut für Radioastronomie, Auf dem Hügel 69, 53121 Bonn, Germany \\ 2 Australia Telescope National Facility, CSIRO, PO Box 76, Epping, NSW 2121, Australia
}

Received 24 September 1998 / Accepted 2 April 2001

\begin{abstract}
Spatial and kinematical correlations between the $\mathrm{H}$ I and ${ }^{12} \mathrm{CO}(2-1)$ emission of the southern spiral galaxy NGC 4945 are studied with a common angular resolution of $\sim 23^{\prime \prime}$ (corresponding to $750 \mathrm{pc}$ at $D=6.7 \mathrm{Mpc}$ ) and a velocity resolution of $\sim 7 \mathrm{~km} \mathrm{~s}^{-1}$. The $1.4 \mathrm{GHz}$ continuum emission is also observed. The $\mathrm{H}$ i kinematics yield a galaxy mass of $\sim 1.4 \times 10^{11} M_{\odot}$ within radius $R=380^{\prime \prime}$, with molecular and neutral atomic gas each contributing $\sim 2 \%$, respectively. A central continuum source of size $77^{\prime \prime} 6 \times 3$ ". $4(250 \times 110 \mathrm{pc})$ is enveloped by a molecular cloud of mass $1.5 \times 10^{9} M_{\odot}$ for $R \leq 7^{\prime \prime}$. 5 , and is rapidly rotating with $V_{\text {rot }} \sim 160 \mathrm{~km} \mathrm{~s}^{-1}$. H I emission from the central region at velocities $\left|V-V_{\text {sys }}\right|>200 \mathrm{~km} \mathrm{~s}^{-1}$ may be related to optically detected gas that is believed to trace an outflow directed towards the halo. Nuclear H I absorption at $V-V_{\text {sys }} \sim+80 \mathrm{~km} \mathrm{~s}^{-1}$ suggests inflow towards the centre, that was so far only seen in molecular lines. Hi features at each end of the major axis $\left(|R| \sim 600^{\prime \prime}\right)$ are interpreted as spiral arms that are viewed tangentially and that also cause prominent emission features in the radio continuum, $\mathrm{H}$, and $\mathrm{CO}$ further inside the galaxy. A central elongated region showing non-circular motions is interpreted as a bar which fuels the nuclear starburst. The $\mathrm{H}$ I and CO position-velocity data have been analysed using linear resonance theory, and possible locations of resonances are identified.
\end{abstract}

Key words. galaxies: active - galaxies: individual: NGC 4945 - galaxies: ISM - galaxies: spiral galaxies: starburst - radio lines: galaxies

\section{Introduction}

Studies of the formation and evolution of spiral galaxies require knowledge of their morphological and kinematical properties. Atomic and molecular hydrogen $\left(\mathrm{HI}\right.$ and $\left.\mathrm{H}_{2}\right)$ are the main interstellar gas components of spiral galaxies and provide excellent tracers to elucidate spiral structure and rotation. Since it is difficult to directly observe $\mathrm{H}_{2}$, carbon-monoxide $(\mathrm{CO})$ is commonly used to probe $\mathrm{H}_{2}$ column densities and molecular masses. Whereas $\mathrm{HI}$ is a good tracer for the outer regions of spiral galaxies, the inner regions are often better studied in CO.

The starburst galaxy NGC 4945 is particularly suited for high resolution and high sensitivity studies. Being a member of the Centaurus group, the nearby edgeon galaxy contains a Seyfert 2 nucleus and is classified

Send offprint requests to: R. Wielebinski,

e-mail: rwielebinski@mpifr-bonn.mpg.de

* Based on observations with the Australia Telescope Compact Array. ATCA is funded by the Commonwealth of Australia for operation as a National Research Facility managed by CSIRO.

** Based on observations with the Swedish-ESO submillimeter telescope, ESO/La Silla, Chile. as SB(s)cd or SAB(s)cd (de Vaucouleurs 1964; Braatz et al. 1997). Distance estimates range from $3.8 \mathrm{Mpc}$ (de Vaucouleurs 1964; Bergman et al. 1992) to $8.1 \mathrm{Mpc}$ (Baan 1985). In order to be consistent with other relevant studies, throughout the paper $6.7 \mathrm{Mpc}$ is used.

Although at optical wavelengths the active nucleus is obscured $\left(N_{\mathrm{H}} \gtrsim 10^{24} \mathrm{~cm}^{-2}\right.$; Guainazzi et al. 2000; Madejski et al. 2000), there is evidence for a nuclear superwind (Chen \& Huang 1997; Lipari et al. 1997) and, at $\lambda=100 \mu \mathrm{m}$, the central region is one of the three brightest IRAS point sources beyond the Magellanic Clouds (IRAS 1989). The nucleus is well-defined at radio frequencies $\left(S_{1.4 \mathrm{GHz}}=4.9 \mathrm{Jy}\right.$; Elmouttie et al. 1997) and shows little ultra compact structure on a milliarcsec scale (Preston et al. 1985; Sadler et al. 1995). Microwave transitions of molecules have been detected in dense clouds that envelope the nucleus; this includes the first discovered "megamaser" (Dos Santos \& Lépine 1979; Batchelor et al. 1982; Whiteoak \& Gardner 1986), a possible circumnuclear disk of radius $0.3 \mathrm{pc}$ with a binding mass of $\sim 10^{6} M_{\odot}$ (Greenhill et al. 1997), and numerous transitions at $\mathrm{cm}$ and $\mathrm{mm}$ wavelengths (see e.g. Whiteoak 1986; Henkel et al. 1990, 1994; Curran et al. 2001). $\mathrm{CO}$ studies suggest the presence of a nuclear gas ring 
Table 1. Properties of NGC 4945 (9 mag, SB(s)cd).

\begin{tabular}{|c|c|c|c|}
\hline Position & $\alpha(2000)$ & $13^{\mathrm{h}} 05^{\mathrm{m}} 27^{\mathrm{s}} \cdot 4$ & \\
\hline of nucleus & $\delta(2000)$ & $-49^{\circ} 28^{\prime} 05^{\prime \prime}$ & 1,2 \\
\hline Distance $^{\text {a) }}$ & $D$ & $6.7 \mathrm{Mpc}$ & 3 \\
\hline Diameter & $D_{25 \mathrm{~m}}$ & $17^{\prime}$ & 4 \\
\hline Systemic & $V_{\text {hel }}$ & 563 and $561 \pm 4 \mathrm{~km} \mathrm{~s}^{-1}$ & 5,7 \\
\hline velocity $^{\mathrm{b})}$ & $V_{\mathrm{lsr}}$ & $555 \mathrm{~km} \mathrm{~s}^{-1}$ & 6 \\
\hline Inclination & $i$ & $78 \pm 3^{\circ}$ & 6,7 \\
\hline Position angle & $P A$ & $43 \pm 1^{\circ} ; 45 \pm 2^{\circ}$ & 6,7 \\
\hline Turn-over radius & $R_{v_{\max }}$ & $5 ! 45 ; 6.6$ & 6,7 \\
\hline Turn-over-velocity & $V_{\max }$ & 165 and $183 \pm 4 \mathrm{~km} \mathrm{~s}^{-1}$ & 6,7 \\
\hline Total mass & $M_{\mathrm{T}}$ & 8.8 and $15 \times 10^{10} M_{\odot}$ & 6,7 \\
\hline
\end{tabular}

a) The value of $6.7 \mathrm{Mpc}$ was chosen for consistency with previous $\mathrm{HI}$ and $\mathrm{CO}$ studies; at this distance $23^{\prime \prime}$ corresponds to $750 \mathrm{pc}$. Evidence for $D=4 \mathrm{Mpc}$ has been summarized by Bergman et al. (1992). If this value were used, gas masses and luminosities have to be multiplied by a factor of 0.36 except for dynamical masses, for which a factor of 0.6 is appropriate. b) $V_{\mathrm{hel}}=V_{\mathrm{lsr}}+4.7 \mathrm{~km} \mathrm{~s}^{-1}$.

References:

1) Whiteoak \& Bunton (1985)

2) Elmouttie et al. (1997)

5) Whiteoak et al. (1990)

4) de Vaucouleurs et al. (1991)

(Whiteoak et al. 1990; Bergman et al. 1992; Dahlem et al. 1993). Properties of NGC 4945 relevant to this paper are summarized in Table 1.

Early optical spectroscopic observations suggest that the rotational velocity increases linearly out to a radius of $r=360^{\prime \prime}$ but also show some evidence of non-circular motions (Peterson 1980; Carranza \& Agüero 1983). Studies of the disk radio continuum, $\mathrm{H}$, and $\mathrm{CO}$ emission have been made by Elmouttie et al. (1997); Ables et al. (1987); and Dahlem et al. (1993) respectively. The large-scale radio emission has a steep (frequency) spectral index $(\alpha=-1.1 \pm 0.1)$; no emission was detected from the halo. H I was found to extend out to $r=7^{\prime}$ with an additional feature at $10^{\prime}$ in the south-west. $\mathrm{CO}(1-0)$ emission is concentrated towards the nucleus, but was also detected out to $6^{\prime}$ along the major axis.

We have used the Compact Array of the Australia Telescope National Facility (ATCA, see Frater \& Brooks 1992) to study the $1.4 \mathrm{GHz}$ continuum and $\mathrm{H}$ I emission of NGC 4945 in more detail and with higher sensitivity than before. We have also observed $\mathrm{CO}(2-1)$ emission using the Swedish-ESO Submillimetre Telescope (SEST; see Booth et al. 1989). In addition to the results presented by Dahlem et al. (1993) our $\mathrm{CO}(2-1)$ data are not confined to the nuclear region but also include significant parts of the disk. H I and CO have been compared at a common angular resolution of $\sim 23^{\prime \prime}$ and channel spacings of 6.6 and $6.3 \mathrm{~km} \mathrm{~s}^{-1}$, corresponding to velocity resolutions of $\sim 7.25$ and $6.5 \mathrm{~km} \mathrm{~s}^{-1}$, respectively; $23^{\prime \prime}$ corresponds to $750 \mathrm{pc}$ at $D=6.7 \mathrm{Mpc}$ (see also Table 1 , footnote "a").

\section{Observations and data reduction}

\subsection{4 GHz continuum emission and HI synthesis data}

The ATCA was equipped with $20 \mathrm{~cm}$ FET receivers with system temperatures between 33 and $55 \mathrm{~K}$. Four observing runs provided 53 independent baselines, and were sufficient to achieve a satisfactory sampling of the $u$ $v$ plane. Table 2 displays the configurations, observing dates and baseline ranges. A standard correlator setup of 512 channels was used with a total bandwidth of $8 \mathrm{MHz}$ centred on $1418 \mathrm{MHz}$. The channel separation was $15.6 \mathrm{kHz}\left(3.3 \mathrm{~km} \mathrm{~s}^{-1}\right)$. An observing cycle was adopted in which a 40-min tracking of NGC 4945 was bracketed by alternate 5-min observations of the phasecalibration sources PKS 1215-457 and PKS 1320-446. The radio galaxy PKS 1934-638 was observed as a flux-density calibrator (assumed to have a flux density of $16.4 \mathrm{Jy}$ at $1.4 \mathrm{GHz}$ ). Observations of the quasar PKS 0407-658 provided antenna spectral bandpasses.

Table 2. ATCA configurations for the H I observations.

\begin{tabular}{llccc}
\hline Config. & Date & \multicolumn{3}{c}{ Baselines $[\mathrm{m}]$} \\
\hline 1.5 B & Oct. 18, 1991 & 31 & to & 4301 \\
1.5 C & Dec. 3, 1991 & 77 & to & 4500 \\
$0.75 \mathrm{C}$ & Aug. 22,1992 & 46 & to & 5020 \\
6.0 C & Feb. 12, 1993 & 153 & to & 6000 \\
\hline
\end{tabular}

The data were reduced with an ATNF modification (Killeen 1992) of the Astronomical Image Processing System (AIPS) of the US National Radio Astronomy Observatory. A single data file of correlated $u-v$ data was produced for each observing run. After editing of the spectra obtained with individual baselines for phase and amplitude errors, the central 400 spectral channels were selected. Those channels free of H I contributions were averaged in the $u-v$ domain for each of the four data sets and merged into a continuum-emission data base. Standard imaging and CLEAN (Högbom 1974) procedures were then applied, and the resultant image was further improved by a standard self-calibration routine (e.g. Cornwell \& Fomalont 1989).

In the $u-v$ domain, the continuum emission was removed from channels containing the H I data using the line-free channels (van Langevelde \& Cotton 1990; Cornwell et al. 1992). After concatenation of the four H I data sets, the gain solutions obtained from the selfcalibration of the continuum image were applied.

To facilitate subsequent analysis two sets of images were produced for both the continuum and line data. "Natural weighting" provided a resolution of $19^{\prime \prime}(\mathrm{RA}) \times$ $25^{\prime \prime}$ (Dec) for investigation of the extended structure; "uniform weighting" yielded a restoring beam of $3.2^{\prime \prime} \times 4^{\prime \prime}$ to reveal the spatial fine structure of the nuclear region. 
A correction for the gain variation across the ATCA 22-m antenna beams was applied to all images.

The rms noise of the final continuum image is $\sim 2.0 \mathrm{mJy}_{\text {beam }}{ }^{-1}$. Faint artifacts are present with flux densities of $5-8 \mathrm{mJy}_{\text {beam }}{ }^{-1}$. The peak-to-noise ratio for the nucleus is of the order of 800 . Averaging two contiguous channels (this yields a channel spacing of $6.6 \mathrm{~km} \mathrm{~s}^{-1}$ ) the rms noise of the $\mathrm{H}$ I data becomes $1.2 \mathrm{mJy}_{\text {beam }}{ }^{-1}$.

To investigate the possibility of missing extended emission, the galaxy was also mapped with the Parkes 64-m telescope. The total H I flux density integrated over velocity for Parkes observations ( $64 \mathrm{Jy} \mathrm{km} \mathrm{s}^{-1}$ ) was found to be $9 \%$ lower than its ATCA counterpart $\left(70 \mathrm{Jy} \mathrm{km} \mathrm{s}^{-1}\right)$. The difference is within the uncertainty of at least $5 \%$ for each estimate. In summary, the comparison of flux densities provides no evidence for missing emission in the ATCA observations.

\section{2. ${ }^{12} \mathrm{CO} \mathrm{J}=2 \rightarrow 1$ data}

$\mathrm{CO}(2-1)$ line emission was mapped during 1993 March 110 with the SEST at an angular resolution of $23^{\prime \prime}$. A $230 \mathrm{GHz}$ SIS receiver yielded system temperatures of 600 $1300 \mathrm{~K}$ on an antenna temperature $\left(T_{\mathrm{A}}^{*}\right)$ scale. The main beam efficiency was 0.46 . An acousto-optical spectrometer, with 1440 channels and a total bandwidth of $1 \mathrm{GHz}$, provided a channel separation of $0.9 \mathrm{~km} \mathrm{~s}^{-1}$.

A rectangular grid of positions was selected, centred on the nucleus of the galaxy (see Table 1), and with offsets parallel or orthogonal to the major axis at $\mathrm{PA}=43^{\circ}$. The spectra were obtained at intervals of $10^{\prime \prime}$ along the major and minor axes, and $14^{\prime \prime}$ elsewhere. In total, 404 positions were sampled with offsets from the nucleus that ranged from $-370^{\prime \prime}$ to $+360^{\prime \prime}$ parallel to the major axis, and from $-60^{\prime \prime}$ to $+60^{\prime \prime}$ perpendicular to it.

All spectra were obtained using a dual beam-switching mode (switching frequency $6 \mathrm{~Hz}$ ), with a beam throw of $11^{\prime} .7$. The integration time was $4 \mathrm{~min}$ per position and, averaging seven contiguous channels (channel spacing: $6.3 \mathrm{~km} \mathrm{~s}^{-1}$ ), the rms noise ranged between 20 and $45 \mathrm{mK}$ on a $T_{\mathrm{A}}^{*}$ scale. A set of three spectra was preceded by a short calibration observation of a black body "paddle" which provided an intensity conversion to $T_{\mathrm{A}}^{*}$. Periodic continuum observations of the small-diameter nucleus of the radio source $\mathrm{Cen} \mathrm{A}$ at $115 \mathrm{GHz}$ provided antenna pointing corrections. The pointing was also assessed by regularly re-observing the profile shape of the CO spectrum at the central position of NGC 4945. These measurements infer absolute positional uncertainties $<8^{\prime \prime}$ and relative positional errors $<5^{\prime \prime}$.

The data were processed with the CLASS package of the Groupe d'Astrophysique de Grenoble. To facilitate a comparison of $\mathrm{CO}$ with $\mathrm{HI}$, the $\mathrm{CO}$ spectra were transformed into a data cube and transferred to the AIPS software.

\section{Results and discussion}

\subsection{A brief overview}

The distributions of $1.4 \mathrm{GHz}$ continuum emission, integrated $\mathrm{H}$ I emission, and integrated $\mathrm{CO}(2-1)$ emission are shown in Fig. 1, superimposed on an optical image from the UK Schmidt SRC (Science Research Council) survey. The position offsets are relative to $\alpha(2000)=13^{\mathrm{h}} 05^{\mathrm{m}} 27^{\mathrm{s}} .4$, $\delta(2000)=-49^{\circ} 28^{\prime} 05^{\prime \prime}$.

\subsubsection{The radio continuum}

The continuum emission (Fig. 1a) shows a bright smalldiameter nucleus superimposed on an elongated distribution of emission. The map is in reasonable agreement with the $1.4 \mathrm{GHz}$ image of Elmouttie et al. (1997) but shows additional details. The peak flux density of the central source is $4.2 \pm 0.1 \mathrm{Jy}$ beam ${ }^{-1}$ at $\alpha(2000)=13^{\mathrm{h}} 05^{\mathrm{m}} 27^{\mathrm{s}} .3$, $\delta(2000)=-49^{\circ} 28^{\prime} 07^{\prime \prime}$ (the position agrees to within $2^{\prime \prime}$ with those given by Whiteoak \& Bunton 1985 and Elmouttie et al. 1997). The flux density integrated over the central source is $4.6 \pm 0.1 \mathrm{Jy}$.

The emission extends over $11.6^{\prime} \times 3.3^{\prime}$ with the major axis at a position angle $\mathrm{PA} \sim 45^{\circ}$. Northeast of the nucleus a ridge-line runs above the major axis and veers to the east at $R \sim 240^{\prime \prime}$. Southwest of the nucleus the ridge appears to run below the major axis, turning to the west at $R \sim 300^{\prime \prime}$. Faint (5 mJy) curved structures extending out of the disk at $R \sim 150-200^{\prime \prime}$ are residual sidelobes caused by the relatively bright emission at the nucleus (cf. Sect. 2.1).

\subsubsection{The $\mathrm{H}$ I distribution}

In our contour map of the $\mathrm{H}$ I distribution (Fig. 1b) the central region is dominated by $\mathrm{H}$ I absorption against the strong continuum emission of the nucleus. However, no H I absorption was observed against the extended continuum outside this central region. Because of the influence of the absorption, the total integrated flux density discussed in Sect. 2.1 is not representative of the total H I content. In the figure, the peak value of the integrated emission is $\sim 3.8 \mathrm{Jy} \mathrm{km} \mathrm{s}^{-1}$ beam $^{-1}$ (at $\left.R \sim+220^{\prime \prime}\right)$. The H I emission extends over $\sim 22^{\prime} \times 4^{\prime}(43 \times 7.8 \mathrm{kpc})$, showing a stretched "S"-structure. Within this, a ridge of emission extends $13^{\prime}$ across the nucleus at $\mathrm{PA} \sim 43^{\circ}$, whereas the major axis as defined by the $3 \%$ intensity level is at $\mathrm{PA} \sim 50^{\circ}$. At the south-western end of the distribution there is a moderately bright $\mathrm{H}$ I concentration that is extending to the north. At the opposite end of the galaxy the $\mathrm{H}$ I distribution extends to the south.

Figure 2a shows the average H I spectrum for the entire galaxy. The velocity relative to the Local Standard of Rest (LSR) extends from 340 to $770 \mathrm{~km} \mathrm{~s}^{-1}$ and the emission peaks at 400 and $715 \mathrm{~km} \mathrm{~s}^{-1}$. The systemic velocity, as given by the average of the extreme and peak velocities, is $V_{\text {sys }} \sim 555$ and $555-560 \mathrm{~km} \mathrm{~s}^{-1}$, respectively (cf. Table 1). The deep asymmetric central depression 

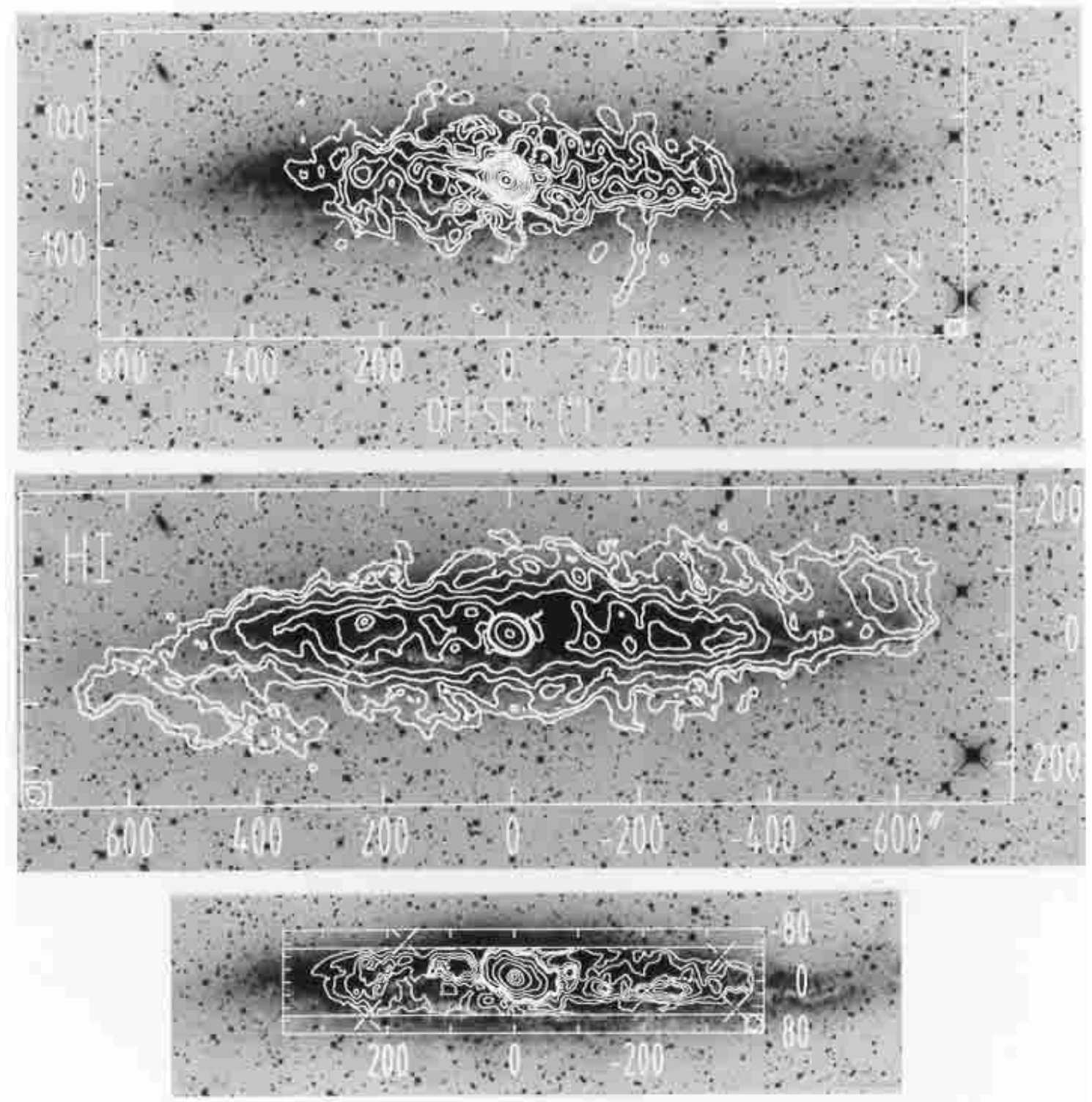

Fig. 1. a) $1.4 \mathrm{GHz}$ continuum superimposed on a UK Schmidt Telescope SRC (Science Research Council; now SERC = Science and Engineering Research Council) J (yellow sensitive emulsion/filter combination) image. The contour levels have flux densities of $5,7.5,10,15,20,25,30,40,60,120,250,500,1000,2000,3000,4200 \mathrm{mJy} \mathrm{beam}^{-1}$. The resolution is $19^{\prime \prime}(\mathrm{RA}) \times 25^{\prime \prime}(\mathrm{Dec})$ (i.e. an average of $\sim 23^{\prime \prime}$ ) along major and minor axes. b) Integrated H I emission superimposed on an SRC-J image. The contours denote $-5000,-500,3,7.5,15,30,50,70,90,99 \%$ of the integrated emission peak of $3.8 \mathrm{Jy} \mathrm{km} \mathrm{s}^{-1}$ beam $^{-1}$. The resolution is as in a). c) Distribution of $\mathrm{CO}(2-1)$ emission integrated over velocity superimposed on a SRC-J image. The contours are 10,20 , $40,80,160,320,640,1280 \mathrm{~K} \mathrm{~km} \mathrm{~s}^{-1}$ beam $^{-1}$ on a $T_{\mathrm{mb}}$ scale. The resolution is $\sim 23^{\prime \prime}$, and the rms noise is $\sim 3 \mathrm{~K} \mathrm{~km} \mathrm{~s}^{-1} \mathrm{beam}^{-1}$. The four white crosses served as reference positions for the overlay.

reflects the presence of absorption against the nucleus. The shape of the spectrum is similar to that obtained with the Parkes telescope directed towards the centre of the galaxy (Whiteoak \& Gardner 1977).

Figure 3a shows our H I spectrum with $\sim 23^{\prime \prime}$ resolution towards the position of the nuclear continuum source. H I is present in absorption against the continuum emission at velocities of $350-770 \mathrm{~km} \mathrm{~s}^{-1}$, i.e. symmetrically offset up to $\sim 210 \mathrm{~km} \mathrm{~s}^{-1}$ from $V_{\text {sys }}$. The profile is asymmetric and appears to be composed of several components. A peak absorption flux density of $1.05 \mathrm{Jy} \mathrm{beam}^{-1}$ is observed at
570 and $635 \mathrm{~km} \mathrm{~s}^{-1}$. The corresponding line-to-continuum ratio is 0.25 .

\subsection{3. $\mathrm{CO}$ emission}

The distribution of $\mathrm{CO}(2-1)$ emission integrated over velocity (Fig. 1c) extends over more than $10^{\prime}$ along the major axis. As the continuum and $\mathrm{HI}$ distributions, $\mathrm{CO}$ also shows a ridge extending above the major axis north-east of the nucleus and below the major axis to the south-west. Like the continuum the CO distribution bends towards the east at $R \sim 250^{\prime \prime}$. 

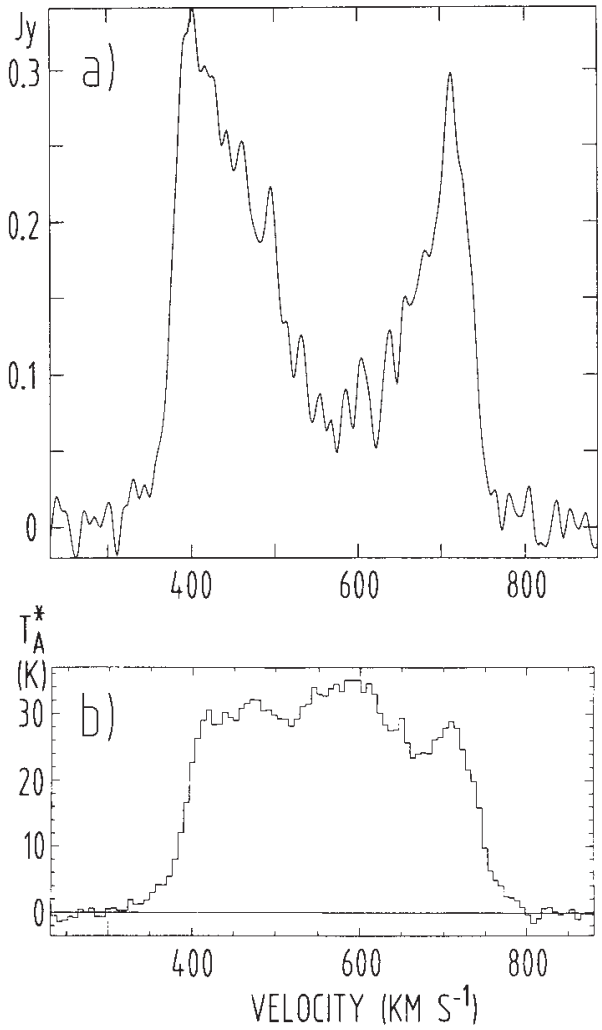

Fig. 2. Line emission profiles as a function of Local Standard of Rest (LSR) velocity of $\mathbf{a}$ ) the $\mathrm{H}$ I and $\mathbf{b}$ ) the $\mathrm{CO}(2-1)$ emission integrated over the observed region with channel spacings of 6.6 and $6.3 \mathrm{~km} \mathrm{~s}^{-1}$, respectively.

Figure $2 \mathrm{~b}$ shows the integrated $\mathrm{CO}(2-1)$ spectrum for the entire region observed. The $\mathrm{CO}$ velocity range is about the same as for the $\mathrm{H}$ I in Fig. 2a. Broad peaks are present near $415,480,580$, and $705 \mathrm{~km} \mathrm{~s}^{-1}$. For an assumed symmetric system, the outer peaks would suggest a systemic velocity of $560 \mathrm{~km} \mathrm{~s}^{-1}$.

Figure $3 \mathrm{~b}$ shows the $\mathrm{CO}(2-1)$ emission towards the nucleus of the galaxy. The $\mathrm{CO}$ covers the same velocity range as the previous spectrum. The profile shape is similar, except that the higher velocity feature is fainter than the systemic feature. In our Fig. 2 and in Fig. 5 of Dahlem et al. (1993), both features have almost the same line temperature. This likely reflects small differences (a few arcsec) in the pointing of the telescope (cf. Sect.2.2). A fitting of Gaussian components yields distinct components centred at velocities of $447,493,593$, and $701 \mathrm{~km} \mathrm{~s}^{-1}$, with a further underlying broad component centred at $\sim 565 \mathrm{~km} \mathrm{~s}^{-1}$.

Figure 4 a shows the integrated $\mathrm{CO}(2-1)$ emission convolved to a resolution of $43^{\prime \prime}$. Combined with corresponding SEST $\mathrm{CO}(1-0)$ data observed at the same resolution (Dahlem et al. 1993), the distribution of the $\mathrm{CO}(2-1) / \mathrm{CO}(1-0)$ ratio is shown in Fig. $4 \mathrm{~b}$. The ratio varies from 0.8 to 2.0 and demonstrates that "warm spots" with ratios larger than unity are not confined to the central region but are also observed far out in the disk. For a possible spatial correlation of these warm molecular regions with spiral arms, see Sect. 3.4.

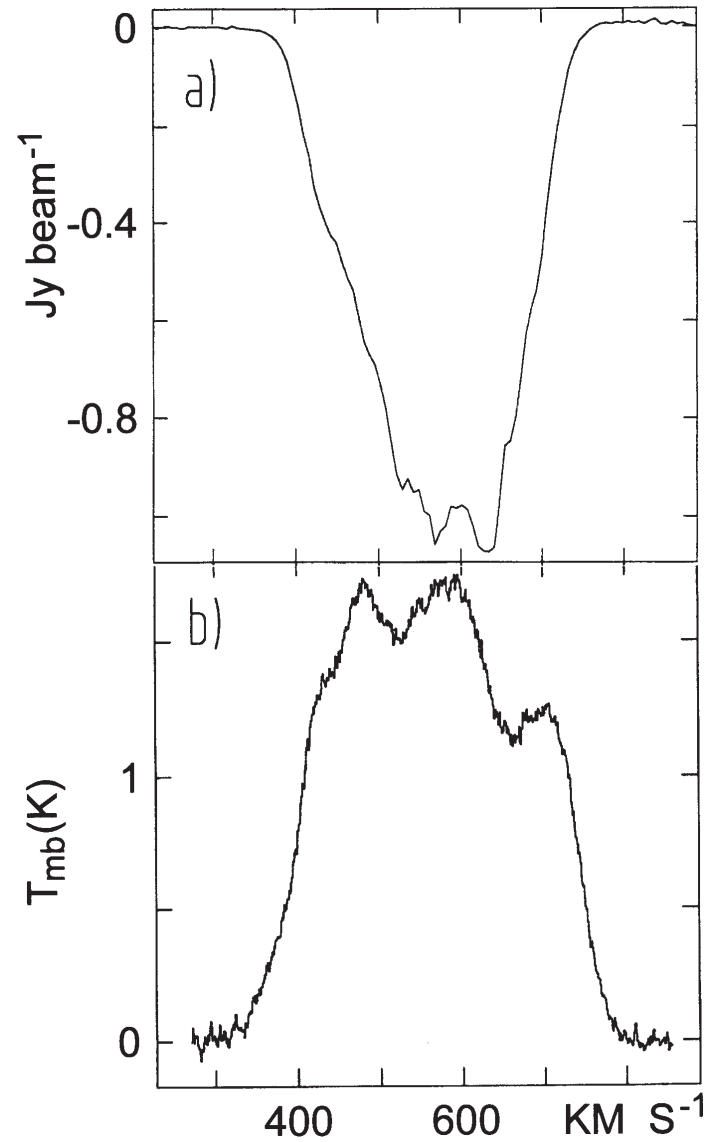

Fig. 3. a) H I spectrum (LSR velocities) towards the central continuum source, obtained with an angular resolution of $19^{\prime \prime}$ $(\mathrm{RA}) \times 25^{\prime \prime}(\mathrm{Dec})$ and a channel spacing of $6.6 \mathrm{~km} \mathrm{~s}^{-1}$. b) The ${ }^{12} \mathrm{CO}(2-1)$ line spectrum towards the centre position with an angular resolution of $23^{\prime \prime}$. The integration time was $60 \mathrm{~min}$ and the channel spacing is $0.9 \mathrm{~km} \mathrm{~s}^{-1}$.

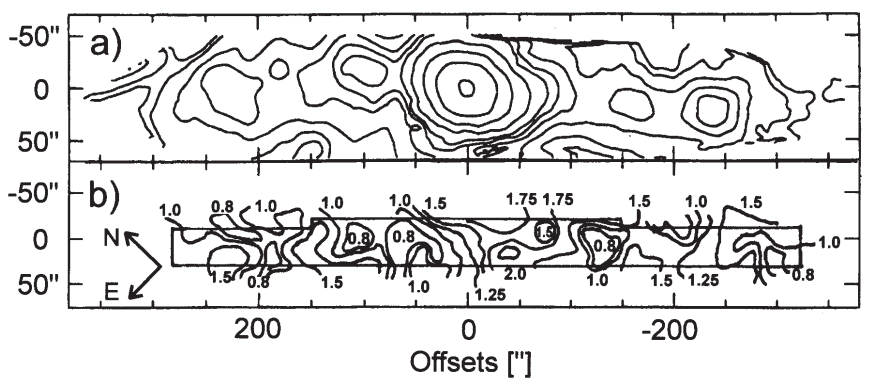

Fig. 4. a) Integrated $\mathrm{CO}(2-1)$ emission convolved to $43^{\prime \prime}$. Contours are $5,10,20,30,40,50,80,160,320,640 \mathrm{~K}$ beam $^{-1}$ on a $T_{\mathrm{mb}}$ scale. b) The $\mathrm{CO}(2-1) / \mathrm{CO}(1-0)$ ratio (in units of $\left.T_{\mathrm{mb}}\right)$ across the disk. The inner box denotes the approximate boundary for "reasonably" convolved $\mathrm{CO}(2-1)$ spectra.

\subsection{The central region}

The strong H I absorption against the central radio continuum source complicates a direct comparison of $\mathrm{H}$ I and CO. The H I absorption must originate from in front of the continuum whereas the $\mathrm{CO}$ emission may arise from in front of and behind the nucleus. This difference is consistent with the $\mathrm{CO}$ and $\mathrm{H}$ I lineshapes shown in Fig. 3, 
where the $\mathrm{CO}$ profile is wider than its H I counterpart at the half-maximum-intensity points. This effect must be significant: We could also plot, instead of the H I absorbing flux, the H I optical depth $\tau(\mathrm{HI})=-\ln \left(1+S_{\mathrm{L}} / F S_{\mathrm{c}}\right)$ with $F$ denoting the continuum source covering factor and $S_{\mathrm{L}}$ and $S_{\mathrm{c}}$ being the (negative) line and (positive) continuum flux. For $F=1$ the profile would resemble that shown in Fig. 3a, since the line remains optically thin even at the line centre. With $F \sim 0.25$, however, H I optical depths would be large near $V \sim 600 \mathrm{~km} \mathrm{~s}^{-1}$ and the H I column density profile would become narrower. The velocity of the central CO peak lies between the velocities of the two strongest $\mathrm{H}$ I absorption components.

Figure 5a shows the nuclear continuum emission at a resolution of $3 . ! 2$ in RA and $4 . ! 0$ in Dec. It is spatially resolved: a Gaussian fit and a correction for beam size yields a source size of 7 "' $6 \times 3$ ". $4( \pm 0$ "! 2$)$ (corresponding to $250 \times 110 \mathrm{pc}$ ), with the major axis at $\mathrm{PA}=42.5$ and a peak intensity of $1.33 \pm 0.03 \mathrm{mJy}_{\text {beam }}{ }^{-1}$. The angular dimensions are reasonably consistent with those $(5 " .7 \times$ $2{ }^{\prime \prime} 0( \pm 0$ ". 1$\left.)\right)$ determined by Whiteoak \& Wilson (1990) at $6 \mathrm{GHz}$. Figures $5 \mathrm{~b}$ and $\mathrm{c}$ show the velocity field of the H I absorption towards the resolved nucleus and the associated position-velocity diagram. The velocity field is centred within $15 \mathrm{kms}^{-1}$ of the galaxy systemic velocity $\left(555-560 \mathrm{~km} \mathrm{~s}^{-1}\right)$ and the velocity gradient across the nucleus is reasonably uniform. It supports the existence of a cloud which surrounds the nucleus and has rapid solidbody rotation. These results are consistent with the $6 \mathrm{GHz}$ OH study by Whiteoak \& Wilson (1990). However, because the $\mathrm{H}$ I absorption is associated only with the region overlying the continuum emission, it cannot provide information about the overall size of the cloud.

The strong $\mathrm{CO}(2-1)$ emission associated with the nucleus (cf. Fig. 1c) has an elongated distribution. Its extent, derived from a Gaussian fit and corrected for beam size, is $37^{\prime \prime} \times 21^{\prime \prime}$ with $\mathrm{PA}=13^{\circ}$. Table 3 contains a comparison with results for $\mathrm{CO}(1-0)$ emission (Dahlem et al. 1993) and $\mathrm{CO}(3-2)$ emission (Mauersberger et al. 1996a). The emission associated with the highest-state $J=3-2$ transition is more centrally concentrated than for the lowerexcitation transitions.

Figure 6 shows the $\mathrm{CO}(2-1)$ position-velocity diagram taken along the major axis. Like Fig. 5c, it supports the presence of a molecular cloud centred on the nucleus and rotating as a solid body with a rotational velocity that reaches $V \sim 160 \mathrm{~km} \mathrm{~s}^{-1}$. The difference between $\left|V-V_{\text {sys }}\right| \sim 160 \mathrm{kms}^{-1}$ and the edge of the H I absorption, $\left|V-V_{\text {sys }}\right| \sim 210 \mathrm{~km} \mathrm{~s}^{-1}$ (Sect. 3.1.2), is likely reflecting the internal linewidth of molecular clouds, the presence of excentric orbits, radial motions caused by cloud collisions, or non-axisymmetric distortions of the gravitational potential (see e.g. Mauersberger et al. 1996b and Sect. 3.5.5). Radius (7".5; taken from Fig. 5) and rotational velocity $\left(\sim 160 \mathrm{~km} \mathrm{~s}^{-1}\right)$ are compatible with those obtained for the $\mathrm{CO}(1-0)$ and $\mathrm{CO}(3-2)$ rings observed by Dahlem et al. (1993) and Mauersberger et al. (1996a), respectively. Bergman et al. (1992) were the first to model the nuclear
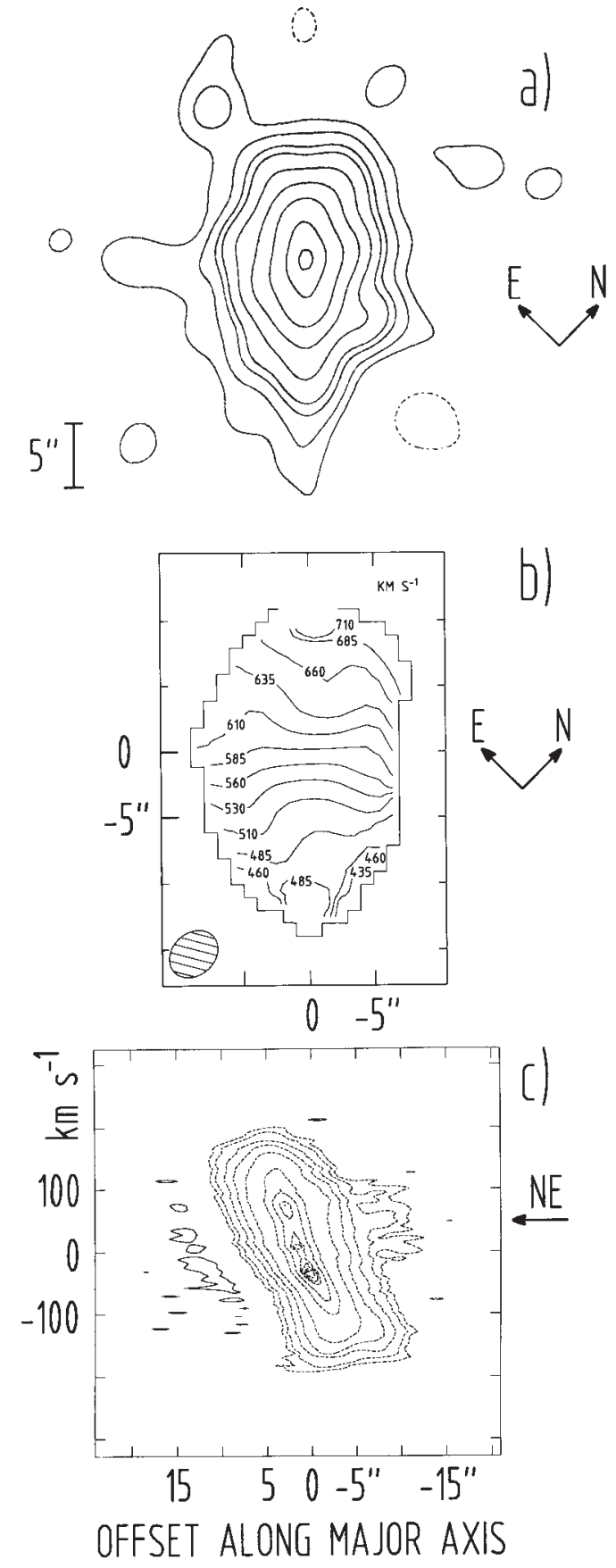

Fig. 5. The resolved nucleus at a resolution of $3.2^{\prime \prime}$ in RA and $4{ }^{\prime \prime} 0$ in Dec. a) The nuclear $1.4 \mathrm{GHz}$ continuum. The contour levels have flux densities of -2 (dashed), 10, 20, 40, 60, 120, 240, $500,1000,1300 \mathrm{mJy}^{-1}$ beam $^{-1}$. b) The LSR velocity field of the nuclear Hi absorption with iso-velocity contours of 435, 460, $485, \ldots 685$, and $710 \mathrm{~km} \mathrm{~s}^{-1}$. The vertical direction is equivalent to $\mathrm{PA}=43^{\circ}$. c) Position-velocity diagram of the nuclear $\mathrm{H}$ I absorption along $\mathrm{PA}=43^{\circ}$ at $\sim 3.6^{\prime \prime} \times 7.25 \mathrm{~km} \mathrm{~s}^{-1}$ resolution. The velocity axis is labeled with respect to $560 \mathrm{~km} \mathrm{~s}^{-1}$. Contours are $-1,1,2,5,10,20,50,70,85,90,95,99 \%$ of the peak absorption of 612 mJy (for the peak flux density in a $\sim 23^{\prime \prime}$ beam, see Fig. 3a).

ring. Henkel et al. (1994) and Curran et al. (2001) commented that nuclear gas densities significantly higher than the originally proposed value $\left(150 \mathrm{~cm}^{-3}\right)$ are required. 
Table 3. Extent of the nuclear CO emission. A Gaussian beam shape and a 2-dimensional Gaussian approximation for the molecular cloud distribution have been assumed.

\begin{tabular}{lccccc}
\hline $\begin{array}{l}{ }^{12} \mathrm{CO} \\
\text { line }\end{array}$ & $\begin{array}{c}\text { Apparent } \\
F W H M\end{array}$ & Beam & \multicolumn{2}{c}{ Deconvolved } & Ref. \\
& {$\left[{ }^{\prime \prime}\right]$} & $\left.{ }^{\prime \prime}\right]$ & {$\left[^{\prime \prime}\right]$} & {$[\mathrm{pc}]$} & \\
\hline$(1-0)$ & $54 \pm 2$ & 43 & $33 \pm 4$ & $1100 \pm 130$ & 1 \\
$(2-1)$ & $32 \pm 2$ & 24 & $21 \pm 4$ & $680 \pm 130$ & 1 \\
& $44 \pm 1$ & 23 & $37 \pm 4$ & $1210 \pm 30$ & $2^{(a)}$ \\
& $31 \pm 1$ & 23 & $21 \pm 4$ & $680 \pm 30$ & $2^{(b)}$ \\
$(3-2)$ & $22 \pm 2$ & 15 & $12 \pm 3$ & $360 \pm 130$ & 3 \\
\hline
\end{tabular}

(1) Dahlem et al. (1993),

(2) This work; ${ }^{(a)}$ major and ${ }^{(b)}$ minor axis values with respect to $\mathrm{PA}=13^{\circ}$,

(3) Mauersberger et al. (1996a), adjusted for $D=6.7 \mathrm{Mpc}$.

Furthermore, the central $\mathrm{CO}(2-1) / \mathrm{CO}(1-0)$ ratio is a factor of two higher than that presumed by Bergman et al. (1992).

The position-velocity diagrams (Figs. 5c and 6) show that for $R<6^{\prime \prime}$, the cloud rotates like a solid body, but at $R>10^{\prime \prime}$ the rotational velocity decreases progressively. If this velocity variation is interpreted in terms of rotation around a central mass confined essentially within $R=7^{\prime \prime} .5$, then the estimated mass is $1.5 \times 10^{9} M_{\odot}$. This is consistent with a value of $0.8-1.6 \times 10^{9} M_{\odot}$ within a radius of $R \sim 3^{\prime \prime}$, as suggested by Koornneef (1993). Mauersberger et al. (1996a) estimated a dynamical mass that, scaled to a galaxy distance of $6.7 \mathrm{Mpc}$, increases from $1.4 \times 10^{9} M_{\odot}$ within $R=5^{\prime \prime} .5$ to $\sim 5 \times 10^{9} M_{\odot}$ within $R=33^{\prime \prime}$.

Figures 7a,b show H I position-velocity diagrams at two position angles $\left(49^{\circ}\right.$ and $\left.43^{\circ}\right)$ to best represent the $\mathrm{HI}$ motions along the major axis. A noteworthy feature is $\mathrm{H}$ I emission from the central region at velocities above $>200 \mathrm{kms}^{-1}$ relative to $V_{\text {sys }}$, and higher than the velocities seen in $\mathrm{H}$ I absorption or $\mathrm{CO}$ emission in Fig. 3. This H I emission (there is no counterpart at $V-V_{\text {sys }}<$ $-200 \mathrm{~km} \mathrm{~s}^{-1}$ ) may be related to an optical outflow from the centre region towards the halo, first suggested by Nakai (1989) on the basis of optical observations. Spectra of the $\mathrm{H} \alpha$ and $[\mathrm{NII}]$ line emission close to the nucleus show high velocities consistent with a conical outflow of gas (Heckman et al. 1990).

\subsection{The overall gas distribution}

Qualitatively, it appears that, in spiral galaxies, neutral atomic gas dominates in the outer regions, whereas molecular gas is concentrated towards the inner disk (e.g. Sanders et al. 1984). Generally, the $\mathrm{CO}(1-0)$ line integral is used to estimate the molecular mass content, conventionally expressed as the $\mathrm{H}_{2}$ mass. For NGC 4945, we have used the $\mathrm{CO}(2-1)$ data on the basis that the uncertainty due to variations in the $\mathrm{CO}(2-1) / \mathrm{CO}(1-0)$ ratio (Fig. 4b)

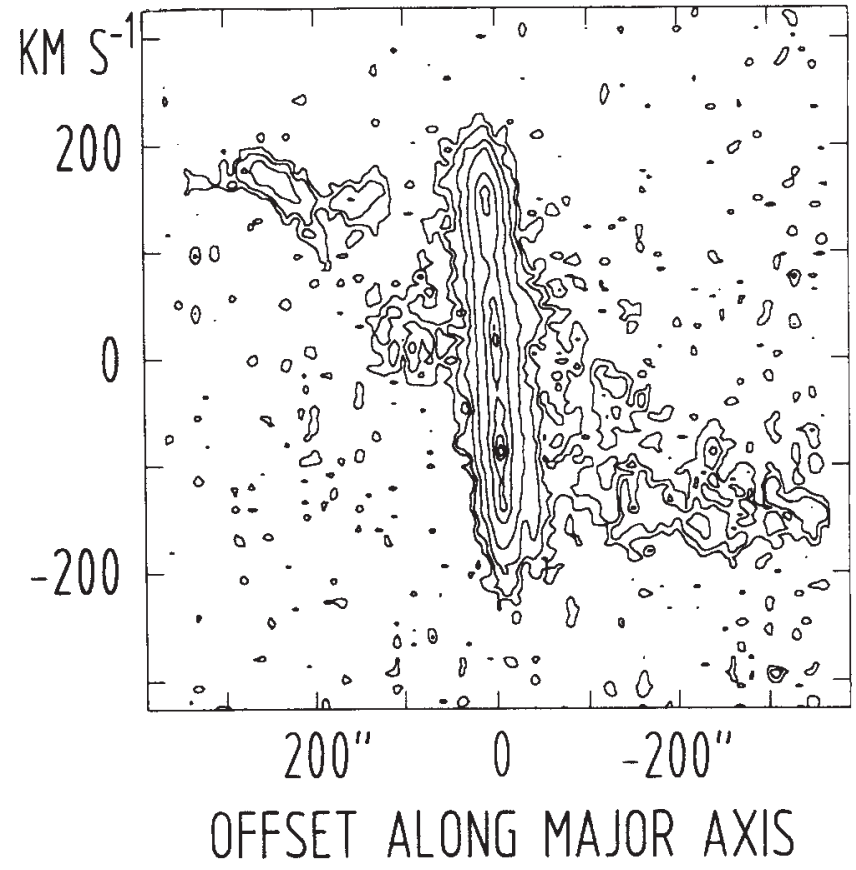

Fig. 6. $\mathrm{CO}(2-1)$ position-velocity diagram of NGC 4945 along the major axis $\left(\mathrm{PA}=43^{\circ}\right)$ with a resolution of $23^{\prime \prime}$ and a channel spacing of $6.3 \mathrm{~km} \mathrm{~s}^{-1}$. The velocity axis is labeled with respect to $560 \mathrm{~km} \mathrm{~s}^{-1}$. Contours are $75,150,300,600,1200$, $1600,1800,1900 \mathrm{mK}$ on a $T_{\mathrm{mb}}$ scale. The rms noise is $\sim 50 \mathrm{mK}$. For the corresponding $\mathrm{CO}(1-0)$ diagram, see Fig. 4 of Dahlem et al. (1993).

is considerably less than the uncertainty in the $\mathrm{CO}$ to $\mathrm{H}_{2}$ conversion factor $X$.

Velocity-integrated $\mathrm{H}$ I and CO line intensities were derived from spectra taken along the major axis between $r=100^{\prime \prime}$ and $360^{\prime \prime}$. The data indicate that the H I column density increases, and the $\mathrm{CO}$ column density decreases, with radius. Using the "standard" conversion factor $X=$ $2 \times 10^{20} \mathrm{~cm}^{-2} /\left(\mathrm{K} \mathrm{km} \mathrm{s}^{-1}\right)$ we find for the inner region, at e.g. $r \sim 135^{\prime \prime}$, that the column density ratio of $\mathrm{HI} / \mathrm{H}_{2}$ is of order 0.2 . At the edge of the observed $\mathrm{CO}(2-1)$ disk, i.e. at $R \sim 320-350^{\prime \prime}$, the $\mathrm{HI} / \mathrm{H}_{2}$ column density ratio becomes $\sim 1$. However, these ratios should be treated with caution, because Mauersberger et al. (1996a,b) presented compelling evidence that, at least for the central regions of NGC 253 and NGC 4945, the value of $X$ is almost an order of magnitude lower than the standard value. Thus the $\mathrm{HI} / \mathrm{H}_{2}$ abundance ratio should also be $\sim 1$ in the inner region.

The velocity-integrated H I emission (Fig. 1b) suggests a variation in major axis position angle and perhaps inclination across the galaxy. As mentioned earlier, there appears to be an $\mathrm{H}$ I inner disk with a lower position angle than that for the outer $\mathrm{H}$ I regions. To investigate whether the galaxy is warped, we modeled the $\mathrm{H}$ I emission with a set of concentric rings (cf. Begeman 1987; Rogstad et al. 1974) of width $50^{\prime \prime}$ from $R=100^{\prime \prime}$ to $650^{\prime \prime}$ and fitted the north-eastern and south-western sides of the galaxy separately. The resulting position angles and inclinations for the rings both vary over less than $10^{\circ}$ along the major 
axis. Part of this variation is systematic in that the position angle varies from $50^{\circ}$ in the north-east to $43^{\circ}$ in the south-west, and for $|R|>400^{\prime \prime}$ the inclination appears about $3^{\circ}$ higher in the south-west than in the north-east. Significant variations in inclination are also found as a function of azimuth and radius in M 31 (see Braun 1991) and may be in part attributable to the presence of spiral arms.

\subsection{Spiral arms}

It has been pointed out already that the distributions of the $1.4 \mathrm{GHz}$ continuum, $\mathrm{HI}$, and $\mathrm{CO}$ in Fig. 1, and also the optical image (for optical images not contaminated by radio contours, see e.g. Nakai 1989; Elmouttie et al. 1997) show a common extended emission ridge which is above the major axis at positive offsets, and below the major axis at negative offsets. There are many emission features associated with the ridge, and the most prominent are listed in Table 4. Several emission peaks are located at common major axis offsets, and are presumably related physically. The $\mathrm{H}$ I distribution shows distinctive features at each end of the galaxy, at offsets of $+620^{\prime \prime}$ and $-580^{\prime \prime}$. They extend away from the major axis and resemble the tangential locations of outer trailing spiral arms of a highly inclined galaxy. At the north-eastern end of the galaxy, which is moving away from us, the arm ending at the near side extends around behind the plane, above the major axis, and joins an inner disk at the location of the emission features at offset $\sim-250^{\prime \prime}$. At the south-western end, rotating towards us, the arm extends around the front of the galaxy, below the major axis, joining the inner disk at the location of the emission peak at offset $+230^{\prime \prime}$. The prominent dust lane present in the optical image would be associated with this arm. This interpretation accounts for the anti-symmetry of the inner emission ridge (see also Dahlem et al. 1993 for the inner part of the galaxy).

It is noteworthy that strong IRAS point sources are located at $R \sim 240^{\prime \prime}$ on each side of the nucleus. At these locations the ${ }^{12} \mathrm{CO}(2-1) /{ }^{12} \mathrm{CO}(1-0)$ line intensity ratio is $>1$. These features are consistent with regions of enhanced star formation, which may reside in spiral arms viewed near their tangential point.

The interpretation could be extended to include the regions of enhanced emission at offsets of about $-120^{\prime \prime}$ and $+120^{\prime \prime}$ as additional tangential locations of the two spiral arms. Then one arm would extend through offsets $+620^{\prime \prime},-250^{\prime \prime}$ and $+120^{\prime \prime}$, and the other through offsets $-580^{\prime \prime},+230^{\prime \prime}$ and $-120^{\prime \prime}$.

Interpreting the distributions in Fig. 1 in terms of a two-arm spiral structure, we can test whether the designated tangential locations are consistent with an overall regular spiral pattern. We therefore consider a spiral described by $R=R_{0} \times \exp a \vartheta$, with the pitch angle $\psi$ in degrees given by $a=\tan \psi$ and the azimuth angle $\vartheta$ in radians. Consideration of the two inner tangential offsets for each arm yields $\psi=12^{\circ}$ and $13^{\circ}$. For a pair of positions
Table 4. Positions of regions with enhanced continuum, H I or $\mathrm{CO}$ emission. Positional errors are $<25^{\prime \prime}$.

\begin{tabular}{ccc}
\hline \multicolumn{3}{c}{ Offsets in arcsec } \\
Cont. & H I & $\mathrm{CO}(2-1)$ \\
\hline & +620 & \\
+230 & +230 & +230 \\
& & +170 \\
+130 & +120 & +90 \\
-55 & & -80 \\
-110 & -120 & -140 \\
-175 & -185 & -195 \\
-215 & -235 & -250 \\
-255 & -270 & \\
-290 & -320 & \\
& -335 & -350 \\
& -580 & \\
\hline
\end{tabular}

consisting of the outermost and central offsets, $\psi=16^{\circ}$ for both arms. The results are plausible - two major spiral arms with pitch angles of about $12-13^{\circ}$ in the inner region and $16^{\circ}$ in the outer region. They are consistent with arms in other spiral galaxies (e.g. Puerari \& Dottori 1992) and might also be detectable by near infrared imaging.

\subsection{Disk kinematics and mass}

\subsubsection{Modelling the overall velocity distribution}

A commonly used relation to describe the rotation curve of a galaxy is

$$
\frac{V}{V_{\max }}=\frac{\frac{R}{R_{v_{\max }}}}{\left(\frac{1}{3}+\frac{2}{3} \times\left(\frac{R}{R_{v_{\max }}}\right)^{n}\right)^{(3 / 2 n)}}
$$

(see Eqs. (26) and (28) of Brandt et al. 1960), where $n$ is a measure of the steepness of the rotation curve and $R_{v_{\max }}$ is the radius at which the maximum rotation velocity $V_{\max }$ occurs. This curve results in solid-body rotation at small radii and a Keplerian-type velocity decrease at large radii. For a flat spiral galaxy inclined to the line-of-sight (such as NGC 4945), the rotation curve can be derived from the variation of radial velocity along the major axis, assuming that the maximum velocity at any position, corrected for the galaxy inclination, represents the tangential (i.e. circular) velocity at the corresponding galactocentric radius.

Inspection of the $\mathrm{H}$ I position-velocity diagrams along the major axis (Figs. 7a,b) suggests the existence of two general velocity regimes. The rapidly rotating central molecular cloud has been discussed already in Sect.3.2. The second system relates to the main disk of the galaxy, viewed at a position angle of $\sim 45^{\circ}$. It extends out to $R \sim$ $400^{\prime \prime}$, with rotational velocities reaching $V \sim 170 \mathrm{~km} \mathrm{~s}^{-1}$ (the correction for an inclination of $78^{\circ}$ is only $2 \%$ and is ignored). This disk is associated with the inner concentration seen in the distribution of integrated H I (Fig. 1b). In the position-velocity diagrams the additional $\mathrm{HI}$ 
Table 5. Parameters derived from the Brandt-curve fits and used to model the large scale velocity field for the H I disk.

\begin{tabular}{lll} 
& & $\mathrm{H} \mathrm{I}$ \\
\hline Kinematical centre & $\alpha(2000)$ & $13^{\mathrm{h}} 05^{\mathrm{m}} 25^{\mathrm{s}} 34$ \\
& $\delta(2000)$ & $-49^{\circ} 29^{\prime} 09^{\prime \prime}$ \\
Systemic velocity & $V_{\text {sys }}$ & $557 \pm 3 \mathrm{~km} \mathrm{~s}^{-1}$ \\
Max. rotation vel. & $V_{\max }$ & $170 \pm 5 \mathrm{~km} \mathrm{~s}^{-1}$ \\
Turn-over point & $R_{v_{\max }}$ & $380^{\prime \prime} \pm 10$ \\
Position angle & $\mathrm{PA}$ & $45^{\circ} \pm 2$ \\
Inclination angle & $i$ & $78^{\circ} \pm 1$ \\
Brandt curve index & $n$ & $3.5 \pm 1$ \\
\hline
\end{tabular}

concentrations at $R \sim 600^{\prime \prime}$ then correspond to the spiral arms that have already been discussed (Sect. 3.4).

Because of the high inclination of the galaxy, a lineof-sight at a specific offset along the major axis will also include $\mathrm{H}$ I at galactocentric radii larger than the radius corresponding to that offset. The line-of-sight velocities of the extra H I components are smaller than the rotational velocity at a specific offset and are responsible for the lowvelocity "tail" present at many positions along the major axis.

Single Gaussian profiles were fitted to individual spectra in the $\mathrm{H}$ I dataset. To avoid the effects of wings in the H I spectra, only the top half of the H I profiles with $S_{\mathrm{L}} \geq S_{\text {peak }} / 2$ was used in the fitting. Applying an iterative procedure, Eq. (1) was fitted to the profile velocities for offsets out to $\pm 450^{\prime \prime}$. The parameters listed in Table 5 were obtained for the disk region. They are consistent with the values obtained from a similar fit to $\mathrm{CO}(1-0)$ data by Dahlem et al. (1993). Our $n$ values are 3 (for $\mathrm{HI}$ ) and, from a similar fit to the $\mathrm{CO}(2-1)$ data, 4 , with small formal errors. Our value for $n$ is significantly smaller than that determined by Ables et al. (1987), $n \sim 7$. This may be a consequence of the better angular resolution of our data. Inclusion of the outermost features in the analysis would have increased $R_{v_{\max }}$; this value is not well-defined in the fit to the $\mathrm{H}$ I position-velocity diagram and the small uncertainty in Table 5 only accounts for the formal errror.

\subsubsection{The mass of NGC 4945}

The mass of NGC 4945 can be derived with

$M=(1.5)^{\frac{3}{n}} \times 1242 \times V_{\max }^{2} \times R_{\max } \times D$

from the parameters in Table 5 (see e.g. Eq. (29) of Brandt 1960; Rogstad et al. 1967; Dahlem et al. 1993). $M$ is in solar masses, $V_{\max }$ is in $\mathrm{kms}^{-1}, R_{\max }$ is in arcsec, and $D$ is in Mpc. For $V_{\max }=170 \mathrm{~km} \mathrm{~s}^{-1}$ and $R_{\max }=380^{\prime \prime}$, $M=1.4 \times 10^{11} M_{\odot}$. Reference to the H I position-velocity diagrams (Fig. 7) suggests that this value may be a lower limit, because the velocities of the outermost $\mathrm{H}$ I features are larger than the values given by the fitted rotation curve. The paucity of $\mathrm{H}$ I at offsets of 400-500" may sig- nify a lack of gas at the tangential location along the linesof-sight, in which case the observed velocities are less than the rotation velocities (see also Sect. 3.5.6).

The total integrated $\mathrm{H}$ I flux density of $70 \mathrm{Jy} \mathrm{km} \mathrm{s}^{-1}$ yields an $\mathrm{H}_{\mathrm{I}}$ mass of $7.5 \times 10^{8} M_{\odot}$, which is only $0.5 \%$ of the total mass and considerably less than expected for such galaxies. However, the presence of the central H I absorption has badly affected the integration. Exclusion of the H I spectrum observed towards the peak of the nuclear continuum emission (see Fig. 3a) would have increased the integrated flux density by more than $200 \mathrm{Jy} \mathrm{km} \mathrm{s}^{-1}$, yielding a total mass fraction of $2 \%$.

Because of the limited coverage of the CO distribution, the molecular content of the galaxy was not estimated from our $\mathrm{CO}(2-1)$ data. Assuming an integrated $\mathrm{CO}$ intensity to $\mathrm{H}_{2}$ column density conversion factor of $X=N\left(\mathrm{H}_{2}\right) / I_{\mathrm{CO}}=2.3 \times 10^{20} \mathrm{~cm}^{-2}\left(\mathrm{~K} \mathrm{~km} \mathrm{~s}^{-1}\right)^{-1}$, Dahlem et al. (1993) derived a molecular gas mass fraction of $5 \%$ from their $\mathrm{CO}(1-0)$ data extending out to $360^{\prime \prime}$. While the $X$-factor is appropriate for the local Galactic disk, it may be too large by a factor of $2-5$ for a galaxy with a strong nuclear contribution to the overall $\mathrm{CO}$ emission (see e.g. Mauersberger et al. 1996a). Thus H I and molecular masses in NGC 4945 should be similar, at the order of $2 \%$ of the total mass inside a radius of $380^{\prime \prime}(12 \mathrm{kpc})$.

\subsubsection{Kinematical fine structure}

To compare the H I and CO kinematics on a small scale, pairs of spectra were taken at offsets between $-100^{\prime \prime}$ and $-360^{\prime \prime}$ along the major axis, but with a $10^{\prime \prime}$ offset to the south; these directions provided the highest CO intensities for comparison. Velocity differences between $\mathrm{H}_{\mathrm{I}}$ and $\mathrm{CO}(2-1)$ features were found to range from 5 to $20 \mathrm{~km} \mathrm{~s}^{-1}$. They are not systematic and show a random variation in sign. Aside from the existence of $\mathrm{H}$ I and $\mathrm{CO}$ clouds with separate peculiar motions, for a highly inclined galaxy such as NGC 4945, differences may arise because a number of clouds at different physical locations within the galaxy are seen simultaneously in one beam. A line-of-sight in a specific direction along the major axis may include clouds located at different galactocentric radii or even at different heights above and below the galactic plane.

In the first case (different galactocentric radii), a lineof-sight displacement of 1.5 to $3 \mathrm{kpc}$ between $\mathrm{H} \mathrm{I}$ and $\mathrm{CO}$ could explain velocity differences of 5 to $20 \mathrm{~km} \mathrm{~s}^{-1}$. For comparison, the CO emission peaks in M51 are typically separated by 1.5 to $2 \mathrm{kpc}$ along the spiral arms (GarcíaBurillo et al. 1993). Much smaller line-of-sight displacements could explain the observed velocity differences if streaming motions due to density waves were prominent (for M 51, see Aalto et al. 1999).

In the second case (different heights above the plane), significantly slower rotation velocities might exist in the halo compared to the underlying disk, as observed in the case of M 82 (Sofue et al. 1992). Also in our Galaxy 


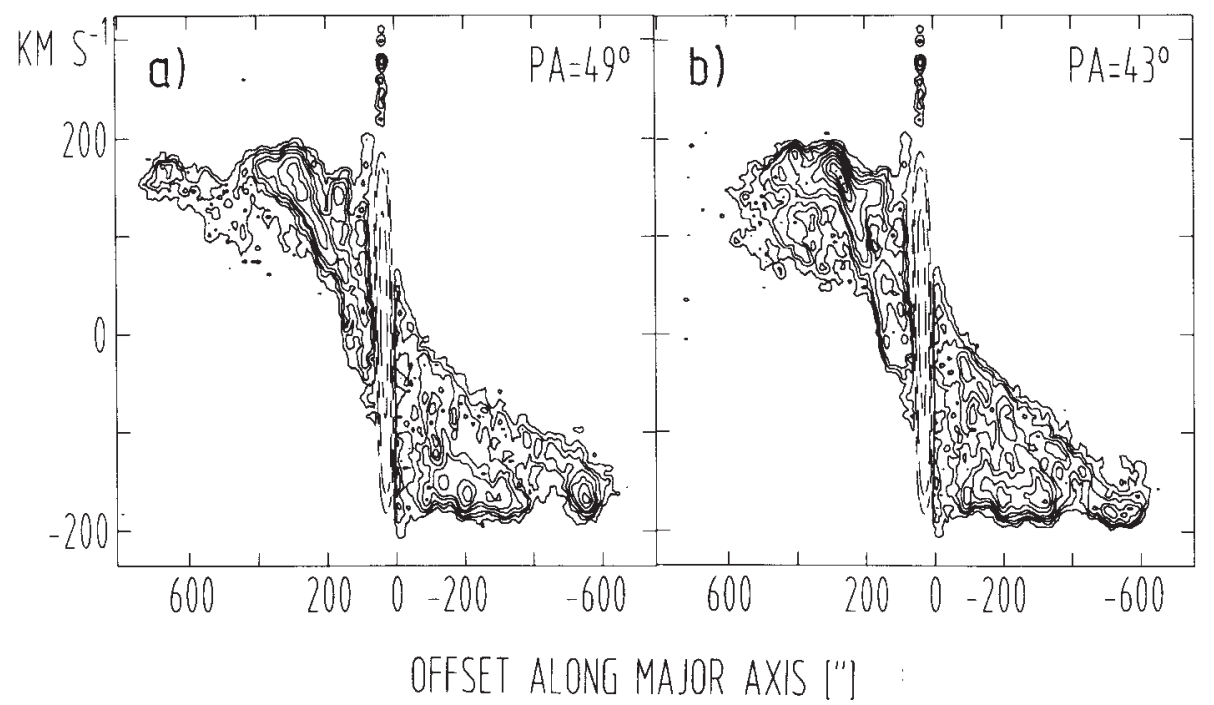

Fig. 7. Position-velocity diagrams of $\mathrm{H}$ I with a resolution of $\sim 23^{\prime \prime} \times 7.25 \mathrm{~km} \mathrm{~s}^{-1}$, obtained along the major axis at a) PA $=49^{\circ}$ and b) $\mathrm{PA}=43^{\circ}$. The velocity axis is labeled with respect to $560 \mathrm{~km} \mathrm{~s}^{-1}$. The contour levels have flux densities of $-500,-100$, $-25,4,7,10,15,20$, plus a) $30,40,50,55$, and b) $30,40,45 \mathrm{Jy} \mathrm{beam}^{-1}$.

peculiar velocities are observed in high-latitude $\mathrm{H}$ I gas (e.g. de Boer 1985; their Fig. 2).

\subsubsection{Velocity fields and velocity residuals}

Figure 8a shows the H I velocity field. The distribution shows anti-symmetric contour distortions with respect to the major axis, indicating departures from uniform circular motion. Considering a given velocity isophote northwest of the major axis, contours are displaced towards the north, whereas south-east of the major axis contours are displaced to the south. The corresponding CO (2-1) velocity field indicates a similarly anti-symmetric behaviour.

Figure $8 \mathrm{~b}$ displays the model velocity field obtained using Eq. (1) and the parameters listed in Table 5. To examine the departure of H I motions from the estimated circular rotation, the model field was subtracted from the observed H I field. Figure 8c shows the distribution of H I residuals. In the outer parts of the galaxy the residuals are typically $0-20 \mathrm{~km} \mathrm{~s}^{-1}$ with no systematic trends, indicating that motions are to first order consistent with uniform circular motion. However, closer to the nucleus, i.e. above the major axis of the galaxy in the north-east and below the major axis in the south-west, higher residuals of up to $\pm 40 \mathrm{~km} \mathrm{~s}^{-1}$ define an elongated region extending across the nucleus to offsets of $+120^{\prime \prime}$ and $-200^{\prime \prime}$. The systematic departures in $\mathrm{H}$ I velocities from circular motion were first identified by Ables et al. (1987), but the lower resolution of their H I data $\left(47^{\prime \prime} \times 73^{\prime \prime}\right)$ led to a more widespread contamination by the nuclear absorption and inhibited the detection of velocity departures as large as $40 \mathrm{~km} \mathrm{~s}^{-1}$.

Ables et al. (1987) detected velocity residuals of 15 to $30 \mathrm{~km} \mathrm{~s}^{-1}$ south-west and east and of -15 to $-30 \mathrm{~km} \mathrm{~s}^{-1}$ west and north-east of the nucleus. This was interpreted in terms of radial motion towards the nucleus. The residuals of our fit are slightly different both with respect to amplitude (as already mentioned) and geometry: In the north-east, north of the major axis, we only detect significant negative velocity residuals, while in the south-west, south of the major axis, large positive residuals are found (Fig. 8c).

Our Fig. 3 shows a prominent $\mathrm{H}$ I feature displaced by about $+80 \mathrm{~km} \mathrm{~s}^{-1}$ from the systemic velocity. Since this feature is seen in absorption against the nuclear continuum source, it signifies neutral atomic gas approaching the galactic centre. From their $\mathrm{CO}(1-0)$ and $(2-1)$ data, Dahlem et al. (1993) find evidence for inflowing low density molecular gas at velocities of $80 \mathrm{~km} \mathrm{~s}^{-1}$. It seems that our nuclear $\mathrm{HI}$ absorption spectrum that separates the systemic from the inflowing velocity component (for a less conclusive spectrum, see Ables et al. 1987) is tracing the neutral atomic part of this kinematical feature as far as it is located in front of the nucleus. Note that this gas component is located inside the nuclear molecular ring (see Table 3; Bergman et al. 1992; Dahlem et al. 1993), at a galactocentric radius of a few hundred pc or less.

\subsubsection{Evidence for a bar}

De Vaucouleurs (1964) suggested that NGC 4945 contains a central bar, which provides a very efficient mechanism to quickly transport matter towards the nucleus while releasing angular momentum outwards. Can such a bar explain the velocity anomalies outlined in Sect. 3.5.4? A classical signature of a bar is an S-shaped distortion in the isovelocity contours of the gas (e.g. Kalnajs 1978; Duval \& Monnet 1985). This is observed in NGC 4945 (see Fig. 8a; Sect. 3.5.4). If a bar is present, the $\mathrm{H}$ I velocity residuals in Fig. 8c could be interpreted in terms of systematic overall gas flows along it, approaching at positive offsets, receding at negative offsets. The orbit velocities in the bar near the nucleus may be estimated from the pv diagrams and 


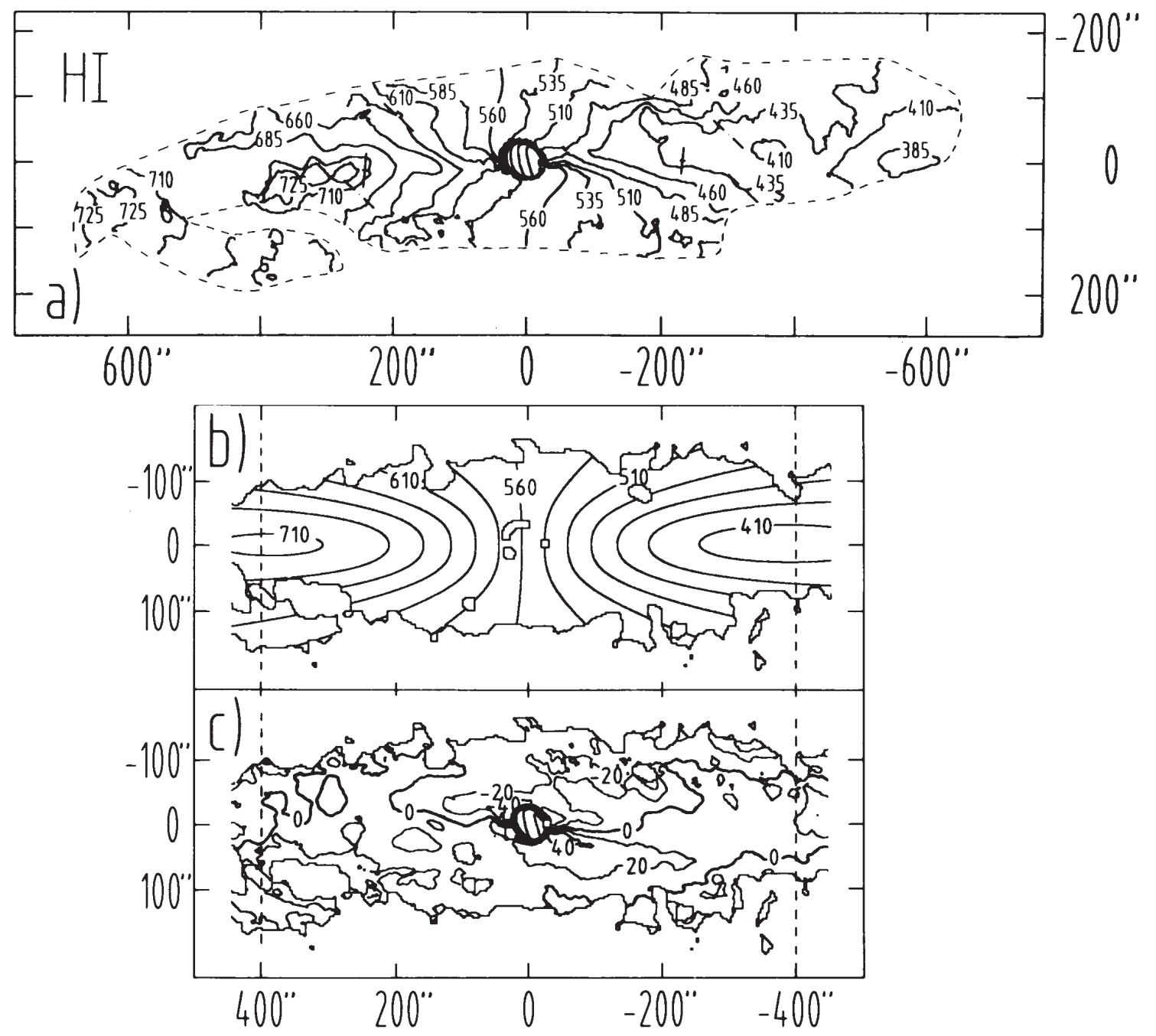

Fig. 8. a) H I velocity field obtained from Gaussian fitting to observed spectra. The iso-velocity contours are at $385,410,435$, $\ldots 710$, and $725 \mathrm{~km} \mathrm{~s}^{-1}$, b) Brandt model velocity field for H I. The contours are spaced at $25 \mathrm{~km} \mathrm{~s}^{-1}$ intervals. c) Residual velocities, after subtraction of the model field from the observed field. The contours are spaced at $20 \mathrm{~km} \mathrm{~s}^{-1}$ intervals.

the velocity field residuals. The continuum and CO distributions in Figs. 1 and 4 show enhanced emission extending out from the nucleus along $\mathrm{PA} \sim 33^{\circ}$, i.e. along the axis that is also showing the velocity anomalies (Fig. 8c). Assuming that the putative bar is associated with the emission ridge introduced above and that this is located within the plane of the galaxy, we obtain an azimuthal angle of $\sim 40^{\circ}$ (counterclockwise) with respect to the lineof-sight projected onto the plane of the galaxy. The total inclination to the line-of-sight would then be $45^{\circ}$.

There exists a strong correlation between the nuclear absorbing column density and the presence of a bar in Seyfert 2 galaxies. Strongly barred Seyfert 2 galaxies have an average $N_{\mathrm{H}}$ that is two orders of magnitude higher than in non-barred Sy 2s. More than $80 \%$ of the "Compton thick Seyfert $2 \mathrm{~s}$ " $\left(N_{\mathrm{H}} \gtrsim 10^{24} \mathrm{~cm}^{-2}\right.$; most of this column density must arise from the innermost few $10 \mathrm{pc}$ ) are barred and almost $60 \%$ of these are "strongly" barred (Maiolino et al. 1999). NGC 4945 is a Compton thick Seyfert 2 galaxy (e.g. Guainazzi et al. 2000; Madejski et al. 2000).
Non-interacting spiral galaxies with moderate inclination and substantial far infrared emission (for the central part of NGC $4945, L_{\mathrm{FIR}} \sim 5 \times 10^{10} L_{\odot}$; IRAS 1989) are known to show both strong and long bars (Martinet \& Friedli 1997). The presence of asymmetric morphologies in individual Seyfert galaxies is positively correlated with their tendency to exhibit enhanced star forming activity (Maiolino et al. 1997). Hence irrespective of direct observational evidence, the presence of a bar in NGC 4945 is, at least statistically, very likely.

The velocity dispersion of $\mathrm{H} \mathrm{I}$ in the central region is also consistent with the presence of a bar. Estimated for each observed H I spectrum during the Gaussian fitting, the disperson in the outer galaxy is $10-30 \mathrm{~km} \mathrm{~s}^{-1}$, which is consistent with the dispersion of gas along the spiral arms in the presence of relatively uniform circular rotation. However, within $\sim 150^{\prime \prime}$ of the nucleus there is an elongated region where the dispersion is above $50 \mathrm{~km} \mathrm{~s}^{-1}$. This may reflect higher gas turbulence or a higher space density of gas clouds near the centre, but could also reflect fast motion on highly eccentric orbits in a bar. 
Since all this evidence is circumstantial, a definite answer to the question whether NGC 4945 has a bar must come from the near-infrared (preferably $K$-band) image. This method proved to be conclusive in the case of another nearby highly inclined southern starburst galaxy, NGC 253 (see e.g. Engelbracht et al. 1998).

\subsubsection{Dynamical analysis}

The $\mathrm{HI}$ and $\mathrm{CO}(2-1)$ pv diagrams can be analysed using linear resonance theory (e.g. Binney \& Tremaine 1987) to deduce the locations of various gravitational resonances within NGC 4945. If we assume the presence of a "weak" bar (the orbits can be described by a superposition of circular motion around the centre and small oscillations caused by the non-axisymmetric part of the potential), the spiral and barred structure is constrained by the locations of these resonances. For a perfectly edge-on barred spiral the locations can be only approximate, because the rotation curve will be affected by the resonances as well as the bar's de-projected size and angle to the line-of-sight. In the case of NGC 4945, the proposed bar (Sect. 3.5.5) is sufficiently displaced from the major axis to provide a reasonable approximation.

For gas orbiting the centre of a galaxy with angular velocity $\Omega=V / R$ at radius $R$, the radial epicyclic frequency $\kappa$ is expressed by

$\kappa(R)=\sqrt{4 \Omega^{2}(R)+R \frac{\mathrm{d} \Omega^{2}(R)}{\mathrm{d} R}}$.

Application of a Brandt rotation curve (Eq. (1)) infers

$\frac{\mathrm{d} \Omega^{2}}{\mathrm{~d} R}=-\frac{2 V_{\max }^{2}}{R_{V_{\max }}^{3}}\left(\frac{R}{R_{V_{\max }}}\right)^{n-1}\left[\frac{1}{3}+\frac{2}{3}\left(\frac{R}{R_{V_{\max }}}\right)^{n}\right]^{\frac{-3-n}{n}}$.

Inner and outer "Lindblad" resonances (ILR and OLR) occur when the pattern speed of the bar $\Omega_{\mathrm{P}}=\Omega-\kappa / m$ and $\Omega+\kappa / m$, respectively ( $m=2$ represents a barred potential, see e.g. Eq. (3-115) of Binney \& Tremaine 1987). Figure 9 shows the measured variation of angular velocity as a function of galactocentric radius. A rotation curve of the form given in Eq. (1) with the parameters outlined in Table 5 has been fitted to $\mathrm{HI}$ velocities derived from the H I pv diagram in Fig. 7. The fitted curve provides a reasonable fit to the outer galaxy ( $R \gtrsim 5 \mathrm{kpc})$.

For a barred spiral galaxy, it is expected that the co-rotation radius $\mathrm{CR}$ would be located not far beyond the end of the bar. For NGC 4945 this position is uncertain. Accounting for unsystematic velocity residuals of $0-20 \mathrm{~km} \mathrm{~s}^{-1}$ (Sect. 3.5.4), the velocity anomaly can be traced out to approximately $\pm 150^{\prime \prime}$ projected on the sky (see Fig. 8c). With an inclination to the line-of-sight of $45^{\circ}$ (Sect. 3.5.5) this would be equivalent to a bar extent (from the nucleus) of about $7 \mathrm{kpc}$. Since the spiral arms could be traced from the outer galaxy $\left(\sim 600^{\prime \prime}\right.$ offset from the centre) to $\sim 120^{\prime \prime}$ (Sect.3.4), the spiral

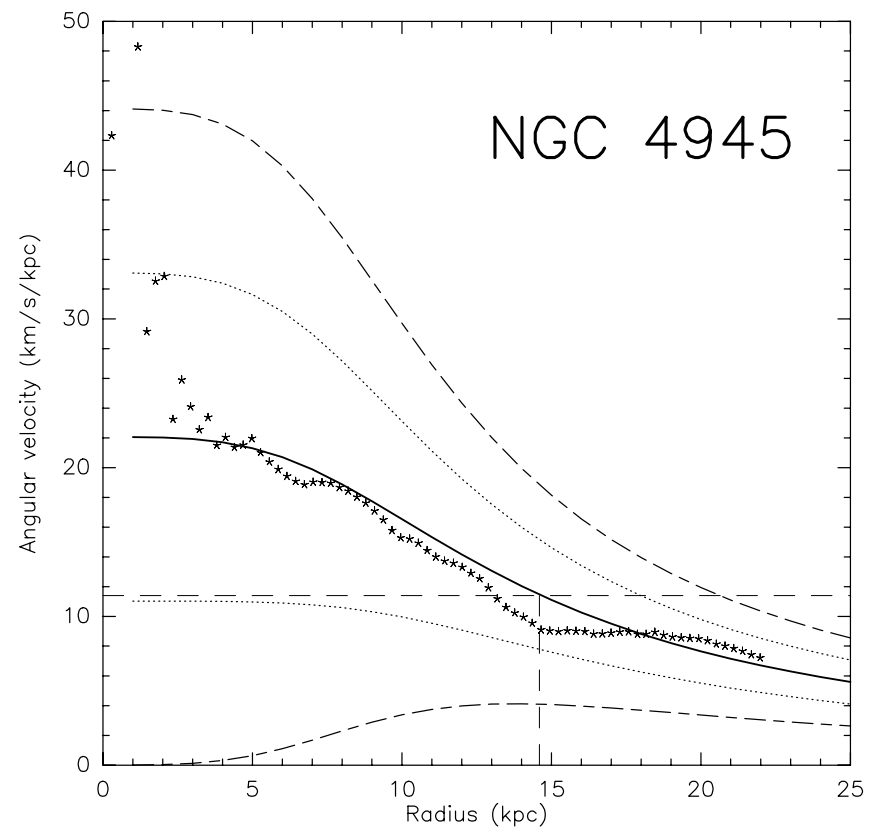

Fig. 9. Variation of angular velocity $(\Omega)$ as a function of galactocentric radius, determined from $\mathrm{H}$ i observations (asterisks; see Sect.3.5.1 for details) and fitted with a Brandt rotation curve (solid line; see Eq. (1)) for the outer disk with parameters given in Table 5 . At $R \sim 15 \mathrm{kpc}$, low measured angular velocities with respect to the fit are likely an artefact caused by a lack of neutral atomic gas at this radius. Shown are also $\Omega-\kappa / m$ and $\Omega+\kappa / m$ for $m=2$ (dashed lines) and $m=4$ (dotted lines). The assumed co-rotation radius (vertical dashed line) and resulting pattern speed $\Omega_{\mathrm{P}}$ (horizontal dashed line) are indicated.

arms might be connected with the bar, as commonly observed in galaxies with small inclination (e.g. Reynaud \& Downes 1997; Hüttemeister et al. 1999). The CR must be located at $R>7 \mathrm{kpc}$, the outer radius of the gaseous bar. Since stellar bars are longer than their gaseous counterparts (Martinet 1995) and since gravitational torques de-populate the co-rotation region in a spiral galaxy (e.g. García-Burillo \& Guélin 1995; Combes 1996), the drop in H I intensity seen in Fig. 7 at $R \sim 450^{\prime \prime}(14.6 \mathrm{kpc})$ and the small "rotational" velocities at this radius (see Fig. 9 and Sect. 3.5.2) may be considered as a signature of the CR (see also Freeman 1997 for "average" radii). Adopting this radius, the pattern speed is $11 \mathrm{~km} \mathrm{~s}^{-1} \mathrm{kpc}^{-1}$; the OLR (which occurs where the pattern speed intercepts the upper $m=2$ curve in Fig. 9) is then at a radius of $21 \mathrm{kpc}$ (angular distance: $\sim 650^{\prime \prime}$ ) and the $m=4$ ultra-harmonic resonance is at $18 \mathrm{kpc}\left(550^{\prime \prime}\right)$, where the outermost $\mathrm{HI}$ features are seen (Fig. 1).

Our Brandt rotation curve is a good fit to the outer galaxy, but does not allow to predict the presence of an ILR. In the innermost parts of the galaxy, measured angular velocities are larger than those suggested by the Brandt curve (Fig. 9), leaving open the possibility that an inner Lindblad resonance exists at $R<3 \mathrm{kpc}$. An attractive but speculative view is to associate the inner molecular ring at a galactocentric radius of a few hundred pc (Table 3) 
with the ILR that might contain a nested secondary bar (the $80 \mathrm{~km} \mathrm{~s}^{-1}$ inflow; see Sect. 3.5.4) guiding atomic and molecular gas to the putative circumnuclear torus discovered by Greenhill et al. (1997). Note however that the nuclear molecular ring is part of the transitional region between solid body and differential rotation. The ring is therefore not necessarily formed by a gravitational resonance. It could also be caused by viscous transport as e.g. outlined by Däther \& Biermann (1990). Adopting the Däther \& Biermann mechanism, an age estimate applying their last equation leads to a formation timescale of $t_{\text {ring }} \sim 1$ Gyr.

\section{Conclusions}

A high-resolution study has been performed of the $1.4 \mathrm{GHz}$ continuum, $\mathrm{HI}$, and $\mathrm{CO}(2-1)$ emission for the southern spiral galaxy NGC 4945. It utilizes both the Australia Telescope Compact Array (ATCA) and the Swedish-ESOSubmillimetre Telescope (SEST). The angular resolution is $\sim 23^{\prime \prime}$ (750 pc at $D=6.7 \mathrm{Mpc}$ ) and the spectral resolution is $\sim 7 \mathrm{~km} \mathrm{~s}^{-1}$. The ATCA results also yield high resolution $\left(\sim 3.6^{\prime \prime}\right)$ images of the nuclear region of the galaxy.

The main conclusions are as follows:

1. The $1.4 \mathrm{GHz}$ continuum emission extends over $11.6^{\prime} \times$ $3.3^{\prime}$ with $\mathrm{PA} \sim 45^{\circ}$ and contains a bright nucleus with a size of $77^{\prime \prime} 6 \times 33^{\prime \prime} 4\left( \pm 00^{\prime \prime} 2\right)$ (corresponding to $250 \times 110 \mathrm{pc}$ ) with the major axis at $\mathrm{PA}=42.5$ and an integrated flux density of $4.6 \pm 0.1 \mathrm{Jy}$, centred at $\alpha(2000)=13^{\mathrm{h}} 05^{\mathrm{m}} 27.33, \delta(2000)=-49^{\circ} 28^{\prime} 07^{\prime \prime}$.

2. Our $\mathrm{CO}(2-1)$ data confirm the presence of a circumnuclear ring at $R \sim 10-20^{\prime \prime}$. H I gas is observed near the centre with a rotation velocity that reaches $160 \mathrm{~km} \mathrm{~s}^{-1}$ at $R=7 " .5(250 \mathrm{pc})$; at smaller and larger radii the velocity is lower. The dynamical mass inside of $7 ! .5$ is $1.5 \times 10^{9} M_{\odot}$.

3. Nuclear H I emission at velocities offset by more than $200 \mathrm{~km} \mathrm{~s}^{-1}$ from the systemic velocity may be related to an outflow towards the halo, observed at optical wavelengths. H I absorption shows evidence for inflowing nuclear gas $\left(\left|V-V_{\text {sys }}\right| \sim+80 \mathrm{~km} \mathrm{~s}^{-1}\right)$ that was previously only seen in $\mathrm{CO}$.

4. $\mathrm{HI}$ is detected beyond the optical $25^{\mathrm{m}} \operatorname{arcsec}^{-2}$ contours. The H I emission extends over $\sim 22^{\prime}(43 \mathrm{kpc})$ at a position angle that increases from $43^{\circ}$ to $50^{\circ}$ from the south-west to the north-east. H I Local Standard of Rest velocities extend from 340 to $770 \mathrm{~km} \mathrm{~s}^{-1}$ and yield a systemic velocity of $555-560 \mathrm{~km} \mathrm{~s}^{-1}$. An analysis of the velocity field indicates that the maximum mean rotation velocity of $170 \mathrm{~km} \mathrm{~s}^{-1}$ occurs at a galactocentric radius of $\gtrsim 380^{\prime \prime}$, yielding a total mass of $1.4 \times 10^{11} M_{\odot}$ inside of $R=380^{\prime \prime}$. Mass fractions of molecular and $\mathrm{H}$ I gas are $\sim 2 \%$ for each component.

5. The H I distribution shows distinctive features at each end of the major axis, offset about $\pm 600^{\prime \prime}$ from the nucleus. These are interpreted as spiral arms viewed tangentially. Other common features in the continuum,
$\mathrm{HI}$ and $\mathrm{CO}$ distributions at offsets near $\pm 120^{\prime \prime}$ and $\pm 240^{\prime \prime}$ may be additional tangential directions for the arms. The results are consistent with two spiral arms having pitch angles of $12-13^{\circ}$ in the inner $\left(|R|<240^{\prime \prime}\right)$ and $16^{\circ}$ in the outer galaxy.

6. The H I velocity field of the galaxy shows a pattern generally consistent with uniform circular motion. Near the nucleus, however, systematic departures exceeding $\pm 30 \mathrm{~km} \mathrm{~s}^{-1}$, S-shaped velocity contours, high infrared luminosities, large nuclear gas column densities, and increased velocity dispersions are interpreted in terms of a bar extending across the nucleus. The data suggest a structure with $\mathrm{PA} \sim 35^{\circ}$, an azimuthal angle of $\sim 40^{\circ}$ (counterclockwise), extending to radial offsets of $\pm 150^{\prime \prime}$ and connecting the inner spiral arms. The likely presence of a bar and large amounts of molecular gas in the inner disk strongly suggest that the starburst in NGC 4945 is ongoing and that a post-starburst stage of evolution is not yet reached.

Acknowledgements. This project was partly supported by the Max-Planck Forschungspreis 1992, awarded to JBW and RW. Discussions with S. Hüttemeister and detailed comments by an anonymous referee are gratefully acknowledged.

\section{References}

Aalto, S., Hüttemeister, S., Scoville, N. Z., \& Thaddeus, P. 1999, ApJ, 522, 165

Ables, J. G., Forster, J. R., Manchester, R. N., et al. 1987, MNRAS, 226, 157

Baan, W. A. 1985, Nature, 315, 26

Batchelor, R. A., Jauncey, D. L., \& Whiteoak, J. B. 1982, MNRAS, 200, 19

Begeman, K. 1987, Ph.D. Thesis, Rijksuniversiteit te Groningen, The Netherlands

Bergman, P., Aalto, S., Black, J. H., \& Rydbeck, G. 1992, A\&A, 265, 403

Binney, J., \& Tremaine, S. 1987, Galactic Dynamics (Princeton University Press, Princeton)

Booth, R. S., Delgado, G., Hagström, M., et al. 1989, A\&A, 216,315

Braatz, J. A., Wilson, A. S., \& Henkel, C. 1997, ApJS, 110, 321

Brandt, J. C. 1960, ApJ, 131, 293

Braun, R. 1991, ApJ, 372, 54

Carranza, G. J., \& Agüero, E. L. 1983, Observatory, 103, 257

Chen, Y., \& Huang, J.-H. 1997, ApJ, 479, L23

Combes, F. 1996, in Barred Galaxies, Proc. IAU Coll. 157, ed. R. Buta, D. A. Crocker, \& B. G. Elmegreen, ASP Conf. Ser., 91, 286

Cornwell, T., \& Fomalont, E. B. 1989, in Synthesis Imaging in Radio Astronomy, ed. R. A. Perley, F. R. Schwab, \& A. H. Bridle, ASP Conf. Ser., 6, 185

Cornwell, T. J., Uson, J. M., \& Haddad, N. 1992, A\&A, 258, 583

Curran, S. J., Johansson, L. E. B., Bergman, P., Heikkilä, A., \& Aalto, S. 2001, A\&A, 367, 457

Dahlem, M., Golla, G., Whiteoak, J. B., et al. 1993, A\&A, 270, 29

Däther, M., \& Biermann, P. L. 1990, A\&A, 235, 55 
de Boer, K. S. 1985, Mitteilungen der Astronom. Gesell., 63, 21

de Vaucouleurs, G. 1964, ApJ, 139, 899

de Vaucouleurs, G., de Vaucouleurs, A., Corwin H. G., et al. 1991, Third Reference Catalogue of Bright Galaxies (RC3) (Springer Verlag, New York)

Dos Santos, P. M., \& Lépine, J. R. D. 1979, Nature, 278, 34

Duval, M. F., \& Monnet, G. 1985, A\&AS, 61, 141

Elmouttie, M., Haynes, R. F., Jones, K. L., et al. 1997, MNRAS, 284, 830

Engelbracht, C. W., Rieke, M. J., Rieke, G. H., Kelly, D. M., \& Achtermann, J. M. 1998, ApJ, 505, 639

Frater, R. H., \& Brooks, J. W. (ed.), 1992, Journal of Electrical and Electronics Engineering Australia, vol. 12

Freeman, K. C. 1996, in Barred Galaxies, Proc. IAU Coll. 157, ed. R. Buta, D. A. Crocker, \& B. G. Elmegreen, ASP Conf. Ser., 91,1

García-Burillo, S., \& Guélin, M. 1995, A\&A, 299, 657

García-Burillo, S., Guélin, M., \& Cernicharo, J. 1993, A\&A, 274,123

Greenhill, L. J., Moran, J. M., \& Herrnstein, J. R. 1997, ApJ, 481, L23

Guainazzi, M., Matt, G., Brandt, W. N., et al. 2000, A\&A, 356,463

Heckman, T. M., Armus, L., \& Miley, G. K. 1990, ApJS, 74, 833

Henkel, C., Whiteoak, J. B., Nyman, L.-Å., \& Harju, J. 1990, A\&A 230, L5

Henkel, C., Whiteoak, J. B., \& Mauersberger, R. 1994, A\&A, 284,17

Högbom, J. 1974, A\&AS, 15, 417

Hüttemeister, S., Aalto, S., \& Wall, W. F. 1999, A\&A, 346, 45

IRAS, 1989, in Cataloged Galaxies and Quasars Observed in the IRAS Survey, Version 2, produced by L. Fullmer, C. Lonsdale, JPL D-1932

Kalnajs, A.J. 1978, in Structure and Properties of Nearby Galaxies, IAU Symp 77, ed. E. M. Berkhuijsen, \& R. Wielebinski, 113

Killeen, N. E. B. 1992, Analysis of Australia Telescope Data, AT Manual No. K2, CSIRO ATNF, Epping
Koornneef, J. 1993, ApJ, 403, 581

Lipari, S., Tsvetanov, Z., \& Macchetto, F. 1997, ApJS, 111, 369

Madejski, G., Zycki, P., Done, C., et al. 2000, ApJ, 535, L87

Maiolino, R., Risaliti, G., \& Salvati, M. 1999, A\&A, 341, L35

Maiolino, R., Ruiz, M., Rieke, G. H., \& Papadopoulos, P. 1997, ApJ, 485, 552

Martinet, L., 1995, Fund. Cosm. Phys., 15, 341

Martinet, L., \& Friedli, D. 1997, A\&A, 323, 363

Mauersberger, R., Henkel, C., Whiteoak, J. B., Chin, Y.-N., \& Tieftrunk, A. R. 1996a, A\&A, 309, 705

Mauersberger, R., Henkel, C., Wielebinski, R., Wiklind, T., \& Reuter, H.-P. 1996b, A\&A, 305, 421

Nakai, N. 1989, PASJ, 41, 1107

Peterson, C. J. 1980, PASP, 92, 397

Preston, R. A., Morabito, D. D., Williams, J. G., et al. 1985, AJ, 90, 1599

Puerari, I., \& Dottori, H. A. 1992, A\&AS, 93, 469

Reynaud, D., \& Downes, D. 1997, A\&A, 319, 737

Rogstad, D. H., Rougoor, G. W., \& Whiteoak, J. B. 1967, ApJ, 150,9

Rogstad, D. H., Lockhart, I. A., \& Wright, M. C. H. 1974, ApJ, 193, 309

Sadler, E. M., Slee, O. B., Reynolds, J. E., \& Roy, A. L. 1995, MNRAS, 276, 1373

Sanders, D. B., Solomon, P. M., \& Scoville, N. Z. 1984, ApJ, 276, 182

Sofue, Y., Reuter, H.-P., Krause, M., Wielebinski, R., \& Nakai, N. 1992, ApJ, 395, 126

van Langevelde, H. J., \& Cotton, W. D. 1990, A\&A, 239, L5

Whiteoak, J. B. 1986, PASA, 6, 467

Whiteoak, J. B., \& Bunton, J. D. 1985, PASA, 6, 171

Whiteoak, J. B., \& Gardner, F. F. 1977, AJP, 30, 187

Whiteoak, J. B., \& Gardner, F. F. 1979, PASA, 3, 319

Whiteoak, J. B., \& Gardner, F. F. 1986, MNRAS, 222, 513

Whiteoak, J. B., Dahlem, M., Wielebinski, R., \& Harnett, J. I. 1990, A\&A, 231, 25

Whiteoak, J. B., \& Wilson, W. E. 1990, MNRAS, 245, 665 